PROCEEDINGS OF THE AMERICAN MATHEMATICAL SOCIETY

Volume 127, Number 4, April 1999, Pages 1119-1125

S 0002-9939(99)04619-5

\title{
UNIFORM FACTORIZATION FOR COMPACT SETS OF OPERATORS
}

\author{
R. ARON, M. LINDSTRÖM, W. M. RUESS, AND R. RYAN \\ (Communicated by Theodore W. Gamelin)
}

\begin{abstract}
We prove a factorization result for relatively compact subsets of compact operators using the Bartle and Graves Selection Theorem, a characterization of relatively compact subsets of tensor products due to Grothendieck, and results of Figiel and Johnson on factorization of compact operators. A further proof, essentially based on the Banach-Dieudonné Theorem, is included. Our methods enable us to give an easier proof of a result of W.H. Graves and W.M. Ruess.
\end{abstract}

\section{INTRODUCTION}

The purpose of this note is to obtain a factorization result for relatively compact subsets of the Banach space of all compact weak*-weak continuous linear maps using a Banach space version of Michael's Selection Theorem which Bartle and Graves [BG] proved in the early fifties. Precisely, they showed that if $X$ and $Y$ are Banach spaces and $u$ is a continuous linear map from $Y$ onto $X$, then there exists a continuous map $f: X \rightarrow Y$ such that $f(x) \in u^{-1}(x)$ for all $x \in X$. In addition to the Selection Theorem, our approach is to use Grothendieck's characterization of relatively compact sets in the projective tensor product and factorization results of compact operators through a universal Banach space, due to Johnson [J] and Figiel $[\mathrm{F}]$. We further present a second method of proof for our factorization result, based on the Banach-Dieudonné Theorem. From our main theorem we obtain extensions of results of Graves and Ruess [GR]. Their methods are different and, in our opinion, more complicated. We also obtain a new proof of a result of Toma, characterizing polynomials that are weakly uniformly continuous on bounded sets.

\section{Preliminaries}

Generally, our notation and terminology are standard and we refer to the books [Di] and [G]. For the definition and properties of $\mathcal{L}_{p}$-spaces the reader is referred to $[\mathrm{LT}]$.

Received by the editors July 25, 1997.

1991 Mathematics Subject Classification. Primary 46B07; Secondary 46B28, 46G20, 47A68.

Key words and phrases. Banach spaces, compact factorization, tensor products, Michael's selection theorem, Banach-Dieudonné theorem.

This note was written while the second and the fourth authors were visiting Kent State University to which thanks are acknowledged. The research of Mikael Lindström was supported by a grant from the Foundation of Åbo Akademi University Research Institute.

(C)1999 American Mathematical Society 
$L_{w *}\left(X^{\prime}, Y\right)\left[K_{w *}\left(X^{\prime}, Y\right)\right]$ is the space of all [compact] weak*-weak continuous linear operators with the usual operator norm. There is an isometric isomorphism $K(X, Y) \simeq K_{w *}\left(X^{\prime \prime}, Y\right)\left[W(X, Y) \simeq L_{w^{*}}\left(X^{\prime \prime}, Y\right)\right]$ given by $T \mapsto T^{\prime \prime}$, where $K(X, Y)[W(X, Y)]$ denotes the space of all [weakly] compact operators from $X$ into $Y$. The closed unit ball of a Banach space $X$ is denoted $B_{X} . X_{c}^{\prime}\left[X_{\tau}^{\prime}\right]$ denotes the dual of $X$ endowed with the topology $c\left(X^{\prime}, X\right)\left[\tau\left(X^{\prime}, X\right)\right]$ of uniform convergence on the [weakly] compact subsets of $X . \mathcal{U}_{c}\left[\mathcal{U}_{\tau}\right]$ denotes a $c-[\tau$ - $]$ neighborhood base of zero consisting of closed absolutely convex sets (disks). If $U \in \mathcal{U}_{c}\left[\mathcal{U}_{\tau}\right]$, we denote by $X_{(U)}^{\prime}$ the completion of the quotient of $X^{\prime}$ by the nullspace of $U$, endowed with the norm defined by the Minkowski-functional of $U$. If $C \subset X$ is a closed bounded disk, we denote by $X_{C}$ the span of $C$ in $X$, endowed with the norm given by the Minkowski-functional of $C$.

In $[\mathrm{F}],[\mathrm{J}]$, the authors proved that there is a universal Banach space $Z$ such that every operator $T \in K(X, Y)$ can be factored as $T=v \circ u$, where $u \in K(X, Z)$ and $v \in K(Z, Y)$. In particular, $Z$ can be chosen as $Z=\left(\sum_{W \subset C_{p}} W\right)_{p}, 1 \leq p \leq \infty$, where $W$ runs through the subspaces of $C_{p}$ (and where, as usual, $p=\infty$ is the $c_{0}$-sum).

We can show that $Z$ also serves as a universal factorization space for $K_{w *}\left(X^{\prime}, Y\right)$ operators. One way to see this is by an application of Johnson's factorization methods to the norm closure in $L\left(X^{\prime}, Y\right)$ of the finite-rank, weak*-weak continuous operators. Another way is the following approach, which makes use of [Ru1, Thm. 1.7 (e)]: if $T \in K_{w *}\left(X^{\prime}, Y\right)$, there exists $U \in \mathcal{U}_{c}$ such that $T(U)$ is relatively compact in $Y$. (For details, compare (2) - (6) of the method 2 of proof of Theorem 1 below.) Defining $\tilde{T}: X^{\prime} / U^{-1}(0) \rightarrow Y$ by $\tilde{T}\left(x^{\prime}+U^{-1}(0)\right):=T\left(x^{\prime}\right)$, and extending to $X_{(U)}^{\prime}$ by continuity, $T$ can be decomposed in the following way:

$$
X^{\prime} \stackrel{i d}{\longrightarrow} X_{c}^{\prime} \stackrel{\pi_{U}}{\longrightarrow} X_{(U)}^{\prime} \stackrel{\tilde{T}}{\longrightarrow} Y
$$

Factoring $\tilde{T}$ through $Z$, the conclusion of the above is the following result.

(1) For every pair of Banach spaces $X, Y$, and for every $T \in K_{w^{*}}\left(X^{\prime}, Y\right)$ there are operators $u \in K_{w^{*}}\left(X^{\prime}, Z\right)$ and $v \in K(Z, Y)$ such that $T=v \circ u$.

If $X$ or $Y$ is an $\mathcal{L}_{1}$-space (resp. an $\mathcal{L}_{\infty}$-space), then Randtke [R2] (resp. Dazord [D], cf. also Randtke [R1], Johnson [J]) has shown that every operator in $K(X, Y)$ factors compactly through $l_{1}$ (resp. $c_{0}$ ).

\section{The REsults}

Given Banach spaces $X, Y$, let the universal Banach space $Z$ be as above. According to (1), the continuous, bilinear map

$$
\tau: K_{w^{*}}\left(X^{\prime}, Z\right) \times K(Z, Y) \rightarrow K_{w^{*}}\left(X^{\prime}, Y\right), \tau(u, v)=v \circ u,
$$

is onto. The linearization $\hat{\tau}: K_{w^{*}}\left(X^{\prime}, Z\right) \hat{\otimes}_{\pi} K(Z, Y) \rightarrow K_{w^{*}}\left(X^{\prime}, Y\right)$ of $\tau$ is a continuous linear onto map. Therefore we can apply the Bartle and Graves selection theorem which asserts that there is a continuous map $\sigma: K_{w^{*}}\left(X^{\prime}, Y\right) \rightarrow$ $K_{w^{*}}\left(X^{\prime}, Z\right) \hat{\otimes}_{\pi} K(Z, Y)$ such that $\hat{\tau} \circ \sigma=i d_{K_{w^{*}}\left(X^{\prime}, Y\right)}$. We remark that the linearization argument is necessary if we wish to apply the Bartle and Graves selection theorem. Indeed, C. Fernandez $[\mathrm{Fe}]$ has recently shown that there are continuous bilinear surjections $\tau: X \times Y \rightarrow Z$ between Banach spaces $X, Y$ and $Z$ for which there is no one-sided inverse. 
Theorem 1. Let $X$ and $Y$ be Banach spaces. For every relatively compact subset $H$ of $K_{w^{*}}\left(X^{\prime}, Y\right)$ there exist an operator $u \in K_{w^{*}}\left(X^{\prime}, Z\right)$, a relatively compact subset $\left\{B_{T}: T \in H\right\}$ of $K(Z)$ and an operator $v \in K(Z, Y)$ such that $T=v \circ B_{T} \circ u$ for all $T \in H$.

Proof. Method 1. By continuity, the set $\sigma(H)$ is relatively compact in $K_{w^{*}}\left(X^{\prime}, Z\right)$ $\hat{\otimes}_{\pi} K(Z, Y)$. Now, by Grothendieck [G, p. 51], there exist null sequences $\left(r_{i}\right)$ in $K_{w^{*}}\left(X^{\prime}, Z\right)$ and $\left(s_{i}\right)$ in $K(Z, Y)$ and a relatively compact subset $K$ of $l_{1}$ such that for each $T \in H$ we can write $\sigma(T)=\sum_{i=1}^{\infty} \lambda_{i}^{T} r_{i} \otimes s_{i}$ where $\lambda^{T}=\left(\lambda_{i}^{T}\right) \in K$.

Define $r: X^{\prime} \rightarrow c_{0}(Z)$ by $r\left(x^{\prime}\right)=\left(r_{i}\left(x^{\prime}\right)\right)$. That $r \in K_{w^{*}}\left(X^{\prime}, c_{0}(Z)\right)$ follows directly from $\left\|r_{i}\right\| \rightarrow 0$. For each $T \in H$ define $A_{T}: c_{0}(Z) \rightarrow l_{1}(Z)$ by $A_{T}(z)=$ $\left(\lambda_{i}^{T} z_{i}\right), z=\left(z_{i}\right) \in c_{0}(Z)$. Since

$$
\sum_{i=1}^{\infty}\left\|\lambda_{i}^{T} z_{i}\right\| \leq \sup _{i}\left\|z_{i}\right\| \cdot \sum_{i=1}^{\infty}\left|\lambda_{i}^{T}\right|,
$$

we have $A_{T} \in L\left(c_{0}(Z), l_{1}(Z)\right)$. Now consider the continuous map $A: l_{1} \rightarrow$ $L\left(c_{0}(Z), l_{1}(Z)\right)$ defined by $A(\lambda) z=\left(\lambda_{i} z_{i}\right)$. Since $A(\bar{K}) \supset\left\{A_{T}: T \in H\right\}$, it follows that the subset $\left\{A_{T}: T \in H\right\}$ of $L\left(c_{0}(Z), l_{1}(Z)\right)$ is relatively compact. Now we define a compact operator $s: l_{1}(Z) \rightarrow Y$ by $s(w)=\sum_{i=1}^{\infty} s_{i}\left(w_{i}\right), w=\left(w_{i}\right) \in l_{1}(Z)$. Compactness of $s$ follows from $\left\|s_{i}\right\| \rightarrow 0$. Since $\hat{\tau} \circ \sigma=i d_{K_{w^{*}}\left(X^{\prime}, Y\right)}$, we conclude that $T=\hat{\tau}(\sigma(T))=\sum_{i=1}^{\infty} \lambda_{i}^{T} s_{i} \circ r_{i}$ and so $T=s \circ A_{T} \circ r$. Finally, we factor $r$ and $s$ through $Z$. Thus, there exist operators $u \in K_{w^{*}}\left(X^{\prime}, Z\right), \alpha \in K\left(Z, c_{0}(Z)\right)$, $\beta \in K\left(l_{1}(Z), Z\right)$ and $v \in K(Z, Y)$ such that $r=\alpha \circ u$ and $s=v \circ \beta$. Let $B_{T}=\beta \circ A_{T} \circ \alpha$ for each $T \in H$. Then $\left\{B_{T}: T \in H\right\}$ is a relatively compact subset of $K(Z)$ and $T=v \circ B_{T} \circ u$ for every $T \in H$.

Method 2: The following facts will be needed:

(2) Given any compact disk $C$ in $Y$, there exists another such, $C_{1}$ say, with $C \subset C_{1}$ such that the $C_{1}$-topology of $Y_{C_{1}}$ restricted to $C$ is equal to $\|\cdot\|_{Y} \mid C$. (Simply take $C_{1}=\bigcap_{n=1}^{\infty}\left(n C+(1 / n) B_{Y}\right)$.)

(3) $c\left(X^{\prime}, X\right)$ is the finest locally convex topology on $X^{\prime}$ agreeing with $c\left(X^{\prime}, X\right)$ on all $n B_{X^{\prime}}, n \in \mathbb{N}$. (Banach-Dieudonné Theorem.)

Now, if $H \subset K_{w^{*}}\left(X^{\prime}, Y\right)$ is relatively compact, then

(4) $H\left(B_{X^{\prime}}\right)$ is relatively compact in $Y$.

Since, accordingly, $H^{*}\left(B_{Y^{\prime}}\right) \subset K_{1}$ a compact disk in $X$,

(5) $H\left(K_{1}^{\circ}\right) \subset B_{Y}$.

Let $U=\bigcap_{n=1}^{\infty}\left(n B_{X^{\prime}}+(1 / n) H^{(-1)}\left(B_{Y}\right)\right)$; then $U \in \mathcal{U}_{c}$ by $(3)$ and $(5)$, and

(6) $H(U) \subset K_{2}$ a compact disk in $Y$. (This follows from (4) and $H(U) \subset$ $n H\left(B_{X^{\prime}}\right)+(1 / n) B_{Y}$ for all $n \in \mathbb{N}$, and, actually, is a special case of [Ru1, Theorem $1.7(\mathrm{e})]$.

Choose a compact disk $K$ in $Y$ related to $K_{2}$ according to (2). Then we have:

(7) Every sequence $\left(T_{n}\right)_{n} \subset H$ has a subsequence that is uniformly Cauchy over $U$ with respect to the $K$-topology. (This follows from operator-norm relative compactness of $H$, together with $U \subset n B_{X^{\prime}}+(1 / n) H^{(-1)}\left(B_{Y}\right)$ for all $n \in \mathbb{N}, H(U) \subset$ $K_{2}$ and $K\left|K_{2}=\|\cdot\|_{Y}\right| K_{2}$.)

Now, given $T \in H$, define $\tilde{T}: X^{\prime} / U^{(-1)}(0) \rightarrow Y_{K}$ by $\tilde{T}\left(x^{\prime}+U^{(-1)}(0)\right)=T\left(x^{\prime}\right)$, and extend continuously to $X_{(U)}^{\prime}$. We then have the following uniform factorization for the operators $T \in H$ :

$$
X^{\prime} \stackrel{i d}{\longrightarrow} X_{c}^{\prime} \stackrel{\pi_{U}}{\longrightarrow} X_{(U)}^{\prime} \stackrel{\tilde{T}}{\longrightarrow} Y_{K} \stackrel{i d_{K}}{\longrightarrow} Y
$$


Now, factor both (the compact weak*-weak-continuous map) $\pi_{U} \circ i d$ through $Z$ as $\pi_{U} \circ i d=r \circ u, u \in K_{w *}\left(X^{\prime}, Z\right), r \in K\left(Z, X_{(U)}^{\prime}\right)$, and (the compact map) $i d_{K}$ as $i d_{K}=v \circ s, s \in K\left(Y_{K}, Z\right), v \in K(Z, Y)$, and let $B_{T}=s \circ \tilde{T} \circ r \in K(Z)$. Then, by (7), $\left\{B_{T} \mid T \in H\right\}$ is relatively compact in $K(Z)$, and $T=v \circ B_{T} \circ u, T \in H$. This completes the proof.

The above method 2 of proof also applies to the case of $L_{w *}$-operators, except that the factor space $Z$ may depend on $X$ and $Y$. Specifically, the following result holds.

Proposition 2. Let $X$ and $Y$ be Banach spaces. There exists a reflexive Banach space $Z=Z(X, Y)$ such that, for every relatively compact subset $H$ of $L_{w^{*}}\left(X^{\prime}, Y\right)$, there exist an operator $u \in L_{w^{*}}\left(X^{\prime}, Z\right)$, a relatively compact subset $\left\{B_{T}: T \in H\right\}$ of $W(Z)$ and an operator $v \in W(Z, Y)$ such that $T=v \circ B_{T} \circ u$ for all $T \in H$.

Proof. Given the assumptions of Proposition 2, in method 2 of proof above, statements (2) to (7) hold with $C$ and $C_{1}$ in (2) weakly compact, $c\left(X^{\prime}, X\right)$ in (3) being replaced by $\tau\left(X^{\prime}, X\right)$ (the assertion then following from the fact that $X_{\tau}^{\prime}$ is a gDFspace, cf. [Ru2]), and, in (4) - (6), "compact" being replaced by "weakly compact". Altogether, the corresponding reasoning thus leads to the following uniform factorization of the $T^{\prime} s$ in $H$ :

$$
X^{\prime} \stackrel{i d}{\longrightarrow} X_{\tau}^{\prime} \stackrel{\pi_{U}}{\longrightarrow} X_{(U)}^{\prime} \stackrel{\tilde{T}}{\longrightarrow} Y_{K} \stackrel{i d_{K}}{\longrightarrow} Y
$$

for some $U \in \mathcal{U}_{\tau}$ and some weakly compact disk $K \subset Y$. At this point, let

$$
Z=Z(X, Y):=\left(\sum_{C, U} R_{C, U}\right)_{2} \oplus_{2}\left(\sum_{K} R_{K}\right)_{2},
$$

where $U$ runs through an $X_{\tau}^{\prime}$-neighbourhood base, $C$ runs through the weakly compact disks in $X_{(U)}^{\prime}$, and $K$ runs through the weakly compact disks in $Y$, and the corresponding $R$-spaces are the associated reflexive Banach spaces in the DavisFigiel-Johnson-Pelczynski factorization [Di] for the range spaces $X_{(U)}^{\prime}$ and $Y$, respectively. Applying now the factorizations corresponding to (1.1) and (2.1) to the mappings of (2.2) completes the proof.

Corollary 3. Let $X$ and $Y$ be Banach spaces. For every relatively compact subset $H$ of $K(X, Y)[W(X, Y)]$, there exist an operator $u \in K(X, Z)[W(X, Z)]$, a relatively compact subset $\left\{B_{T}: T \in H\right\}$ of $K(Z)[W(Z)]$ and an operator $v \in K(Z, Y)[W(Z, Y)]$ such that $T=v \circ B_{T} \circ u$ for all $T \in H$. (Here, the spaces $Z$ are those of Theorem 1 and Proposition 2, respectively.)

In the compact case, this corollary can be combined with factorization results through nice spaces for compact operators between special spaces. In the following result, we apply Corollary 3 when $X$ is an $\mathcal{L}_{1}$-space or an $\mathcal{L}_{\infty}$-space and obtain Theorem 2.1 in [GR]. When $Y$ is an $\mathcal{L}_{1}$-space or an $\mathcal{L}_{\infty}$-space, a similar result can be stated.

Corollary 4. Assume that $X$ is an $\mathcal{L}_{1}$-space (resp. an $\mathcal{L}_{\infty}$-space). For every relatively compact subset $H$ of $K(X, Y)$ there exist an operator $p \in K\left(X, l_{1}\right)$ (resp. $p \in K\left(X, c_{0}\right)$ ) and a relatively compact subset $\left\{Q_{T}: T \in H\right\}$ of $K\left(l_{1}, Y\right)$ (resp. of $K\left(c_{0}, Y\right)$ ) such that $T=Q_{T} \circ p$ for all $T \in H$. 
Proof. Assume that $X$ is an $\mathcal{L}_{1}$-space (resp. an $\mathcal{L}_{\infty}$-space). We apply Corollary 3 ; then, as we have pointed out in the preliminaries, $u$ factors compactly through $l_{1}$ (resp. $c_{0}$ ) such that $u=q \circ p$. Put $Q_{T}:=v \circ B_{T} \circ q$ and we are done.

Finally, we show how Corollary 3 yields a new proof of a result of E. Toma [T]. Recall that $\mathcal{P}\left({ }^{n} X\right)$ denotes the space of continuous $n$-homogeneous polynomials on $X$. Each such polynomial $P$ is associated with a unique element $A$ of the space $\mathcal{L}^{s}\left({ }^{n} X\right)$ of $n$-linear, symmetric mappings on $X$, satisfying $P(x)=A(x, \ldots, x)$ for each $x \in X$. The space of $n$-homogeneous polynomials that are weakly uniformly continuous on the unit ball of $X$ is denoted $P_{w u}\left({ }^{n} X\right)$ and the corresponding space of symmetric $n$-linear forms is denoted $\mathcal{L}_{w u}^{s}\left({ }^{n} X\right)$. For each $n$ homogeneous polynomial $P$ there is a linear operator $T_{P}: X \rightarrow \mathcal{L}^{s}\left({ }^{n-1} X\right)$, defined by $T_{P}\left(x_{1}\right)\left(x_{2}, \ldots, x_{n}\right)=A\left(x_{1}, x_{2}, \ldots, x_{n}\right) . P$ belongs to $P_{w u}\left({ }^{n} X\right)$ if and only if the operator $T_{P}$ is compact; furthermore, if $P \in P_{w u}\left({ }^{n} X\right)$, then $T_{P}$ takes its values in the space $\mathcal{L}_{w u}^{s}\left({ }^{n-1} X\right)$ [AP]. The following corollary is shown in [T]; we offer a different proof, based on the above results.

Proposition 5. Let $X$ be a Banach space and $\mathcal{H}_{n}$ a relatively compact subset of the space $K\left(X, \mathcal{L}_{w u}^{s}\left({ }^{n-1} X\right)\right)$. Then there is a compact subset $K^{\prime}$ of $X^{\prime}$ such that for all $T \in \mathcal{H}_{n}$ and all $x \in X,|T(x)(x, \ldots, x)| \leq \sup _{k^{\prime} \in K^{\prime}}\left|k^{\prime}(x)\right|^{n}$.

Proof. We proceed by induction on $n=2,3, \ldots$. Let $\mathcal{H}_{2}$ be a relatively compact subset of the space $K\left(X, X^{\prime}\right)$ of compact linear maps from $X$ to $\mathcal{L}_{w u}^{s}(X, C)=X^{\prime}$. By Corollary 2, there are a Banach space $Z$, a relatively compact subset $\left\{L_{T}: T \in\right.$ $\left.\mathcal{H}_{2}\right\}$ of $K(X, Z)$, and an operator $w \in K\left(Z, X^{\prime}\right)$ such that $T=w \circ L_{T}$ for all $T \in \mathcal{H}_{2}$. Thus, for each $x \in X$ and for each $T \in \mathcal{H}_{\in}$, we have

$$
|T(x)(x)|=\left|w \circ L_{T}(x)(x)\right|=\left|\left\langle L_{T}(x), w^{t}(x)\right\rangle\right|,
$$

regarding $x \in X \subset X^{\prime \prime}$, and so $|T(x)(x)| \leq\left\|L_{T}(x)\right\| \cdot\left\|w^{t}(x)\right\|$. Now,

$$
\left\|L_{T}(x)\right\|=\sup _{z^{\prime} \in B_{Z^{\prime}}}\left|\left\langle L_{T}(x), z^{\prime}\right\rangle\right|=\sup _{z^{\prime} \in B_{Z^{\prime}}}\left|\left\langle x, L_{T}^{t}\left(z^{\prime}\right)\right\rangle\right| \leq \sup _{k^{\prime} \in K_{1}^{\prime}}\left|\left\langle x, k^{\prime}\right\rangle\right|
$$

where $K_{1}^{\prime}=\overline{\left\{L_{T}^{t}\left(z^{\prime}\right): T \in \mathcal{H}_{2}, z^{\prime} \in B_{Z^{\prime}}\right\}}$ is easily seen to be compact. Furthermore,

$$
\left\|w^{t}(x)\right\|=\sup _{z \in B_{Z}}\left|\left\langle w^{t}(x), z\right\rangle\right|=\sup _{z \in B_{Z}}|\langle x, w(z)\rangle|=\sup _{k^{\prime} \in K_{2}^{\prime}}\left|\left\langle x, k^{\prime}\right\rangle\right|,
$$

where $K_{2}^{\prime}=\overline{w\left(B_{Z}\right)}$ is compact. Therefore, if $K^{\prime}=K_{1}^{\prime} \cup K_{2}^{\prime}$, then

$$
|T(x)(x)| \leq \sup _{k^{\prime} \in K^{\prime}}\left|k^{\prime}(x)\right|^{2}
$$

for all $T \in \mathcal{H}_{2}$ and all $x \in X$.

Assume now that the result is true for all $j<n$ and let $\mathcal{H}_{n}$ be a relatively compact subset of the space $K\left(X, \mathcal{L}_{w u}^{s}\left({ }^{n-1} X\right)\right)$. As before, there are a Banach space $Z$, a relatively compact subset $\left\{L_{T}: T \in \mathcal{H}_{n}\right\}$ of $K(X, Z)$, and an operator $w \in K\left(Z, \mathcal{L}_{w u}^{s}\left({ }^{n-1} X\right)\right)$ such that $T=w \circ L_{T}$ for all $T \in \mathcal{H}_{n}$. Thus, for each $x \in X$ and each $T \in \mathcal{H}_{n}$, we have $|T(x)(x, \ldots, x)|=\left|w \circ L_{T}(x)(x, \ldots, x)\right|=$ $\left|\left\langle L_{T}(x), w^{t}(x, \ldots, x)\right\rangle\right| \leq\left\|L_{T}(x)\right\| \cdot\left\|w^{t}(x, \ldots, x)\right\|$, where we are regarding $(x, \ldots, x)$ as an element of $\mathcal{L}_{w u}^{s}\left({ }^{n-1} X\right)^{\prime}$. Hence, for a compact subset $K_{1}^{\prime} \subset X^{\prime},\left\|L_{T}(x)\right\| \leq$ $\sup _{k^{\prime} \in K_{1}^{\prime}}\left|\left\langle x, k^{\prime}\right\rangle\right|$. Next, we have

$$
\left\|w^{t}(x, \ldots, x)\right\|=\sup _{z \in B_{Z}}\left|\left\langle w^{t}(x, \ldots, x), z\right\rangle\right|=\sup _{z \in B_{Z}}|w(z)(x, \ldots, x)| .
$$


Now $\{w(z):\|z\| \leq 1\} \equiv \mathcal{H}_{n-1}$ is a relatively compact subset of $\mathcal{L}_{w u}^{s}\left({ }^{n-1} X\right)$, which by [AP] means that $\mathcal{H}_{n-1}$ is a relatively compact subset of $K\left(X, \mathcal{L}_{w u}^{s}\left({ }^{n-2} X\right)\right)$. By the induction hypothesis, there is a compact subset $K_{2}^{\prime} \subset X^{\prime}$ such that for all $x \in X$ and all $z \in B_{Z},|w(z)(x, \ldots, x)| \leq \sup _{k^{\prime} \in K_{2}^{\prime}}\left|k^{\prime}(x)\right|^{n-1}$. Letting $K^{\prime}=K_{1}^{\prime} \cup K_{2}^{\prime}$, it follows that

$$
|T(x)(x, \ldots, x)| \leq \sup _{k^{\prime} \in K^{\prime}}\left|k^{\prime}(x)\right|^{n}
$$

for all $T \in \mathcal{H}_{n}$ and all $x \in X$, and the result is proved.

Corollary $6([\mathrm{~T}])$. For any $n$, a continuous $n$-homogeneous polynomial $P$ belongs to $\mathcal{P}_{w u}\left({ }^{n} X\right)$ if and only if there is a compact subset $K^{\prime}$ of $X^{\prime}$ such that $|P(x)| \leq$ $\sup _{k^{\prime} \in K^{\prime}}\left|k^{\prime}(x)\right|^{n}$ for all $x \in X$.

Proof. If $P \in \mathcal{P}_{w u}\left({ }^{n} X\right)$, then $T_{P}: X \rightarrow \mathcal{L}_{w u}^{s}\left({ }^{n-1} X\right)$ is compact and the result follows by applying the proposition to $\mathcal{H}_{n} \equiv\left\{T_{P}\right\}$.

Conversely, suppose there exists a compact subset $K^{\prime}$ of $X^{\prime}$ such that $|P(x)| \leq$ $\sup _{k^{\prime} \in K^{\prime}}\left|k^{\prime}(x)\right|^{n}$ for all $x \in X$. Let $J$ be the polar of $K^{\prime}$ in $X$ and let $\pi$ be the canonical mapping of $X$ onto the Banach space $X_{J}$ associated with $J$. Now the dual of $X_{J}$ is $\left(X^{\prime}\right)_{K^{\prime}}$ and it follows that $\pi$ is compact. Hence $\pi$ is weakly uniformly continuous on the unit ball of $X$. But by the assumption $P$ factors through $\pi$. Therefore $P$ is weakly uniformly continuous on the unit ball of $X$.

\section{ADDED IN PROOF}

The authors are indebted to K. Floret for pointing out that in Method 1 of the proof of Theorem 1, the Bartle-Graves selection theorem is not needed. In fact, by the lifting property of quotient mappings for compact sets, there is a compact set $L \in K_{w^{*}}\left(X^{\prime}, Z\right) \hat{\otimes}_{\pi} K(Z, Y)$ such that $\hat{\tau}(L)=\bar{H}$.

\section{REFERENCES}

[AP] R. M. Aron, J. B. Prolla, Polynomial approximation of differentable functions on Banach spaces, J. Reine Angew. Math. 313 (1980), 195-216 MR 81c:41078

[BG] R. Bartle, L. Graves, Mappings between function spaces, Trans. Amer. Math. Soc. 72 (1952), 400-413 MR 13:951i

[D] J. Dazord, Factoring operators through $c_{0}$, Math. Ann. 220 (1976), 105-122 MR 54:8332

[Di] J. Diestel, Sequences and Series in Banach Spaces, Springer, New York, 1984 MR 85i:46020

[Fe] C. Fernandez, A counterexample to the Bartle-Graves selection theorem for multilinear maps, Proc. Amer. Math. Soc. 126 (1998), 2687-2690. CMP 98:13

[F] T. Figiel, Factorization of compact operators and applications to the approximation property, Studia Math. 45 (1973), 191-210 MR 49:1070

[G] A. Grothendieck, Produits tensoriels topologiques et espaces nucléaires, Amer. Math. Soc. Memoirs 16, Providence, RI, 1955 MR 17:763c

[GR] W. Graves, W. Ruess, Representing compact sets of compact operators and of compact range vector measures, Arch. Math. 49 (1987), 316-325 MR 89a:46145

[J] W.B. Johnson, Factoring compact operators, Israel J. Math. 9 (1971), 337-345 MR 44:7318

[LT] J. Lindenstrauss, L. Tzafriri, Classical Banach Spaces, Lecture Notes in Math., vol. 338, Springer, Berlin, 1973 MR 54:3344

[R1] D. Randtke, A structure theorem for Schwartz spaces, Math. Ann. 201 (1973), 171-176 MR 48:4691

[R2] D. Randtke, A compact operator characterization of $l_{1}$, Math. Ann. 208 (1974), 1-8 MR 49:3507

[Ru1] W.M. Ruess, Compactness and collective compactness in spaces of compact operators, J. Math. Analysis Appl. 84 (1981), 400-417 MR 83h:47032 
[Ru2] W.M. Ruess, [Weakly] Compact operators and DF spaces, Pacific J. Math. 98 (1982), 419441 MR 83h:47034

[T] E. Toma, Aplicacoes holomorfas e polinomios $\tau$-continuous, Thesis, Univ. Federal do Rio de Janeiro, 1993

Department of Mathematics, Kent State University, Kent, Ohio 44240

E-mail address: aron@mcs.kent.edu

Department of Mathematics, Åbo Akademi University, Fin-20500 Åbo, Finland

E-mail address: mlindstr@ra.abo.fi

Fachbereich Mathematik, Universität Essen, D-45117 Essen, Germany

E-mail address: mate00@sp2. power.uni-essen.de

Department of Mathematics, University College Galway, Galway, Ireland

E-mail address: Ray.Ryan@UCG.IE 